IFUM 606/FT

hep-ph/9803390

March 1998

\title{
Regge trajectories and quarkonium spectrum from a first principle Salpeter equation
}

\author{
M. Baldicchi and G.M. Prosperi \\ Dipartimento di Fisica dell'Università di Milano, and \\ I.N.F.N., Sezione di Milano, Italy
}

\begin{abstract}
We compute the heavy-heavy, light-light and light-heavy quarkonium spectrum starting from a first principle Salpeter equation obtained in a preceding paper. We neglect spin-orbit structures and exclude from our treatment the light pseudoscalar states which in principle would require the use of the full Bethe-Salpeter equation due to the chiral symmetry breaking problem. For the rest we find an overall good agreement with the experimental data. In particular for the light-light case we find straight Regge trajectories with the right slope and intercepts. The strong coupling constant $\alpha_{\mathrm{s}}$, the string tension $\sigma$ occurring in the potential and the heavy quark masses are taken from the heavy quarkonium semirelativistic fit with only a small rearrangement. The light quark masses are set equal to baricentral value of the current quark masses as reported by the particle data group. For what concerns the lightlight and the light-heavy systems the calculation is essentially parameter free.
\end{abstract}

PACS:12.38.Aw,11.10.St,12.38.Lg,12.39.Ki

Keywords: Quarkonium spectrum, Regge trajectories, Salpeter equation. 
In reference [1] a Bethe-Salpeter like equation was derived which provides in principle a fully relativistic description of the quark-antiquark system. The derivation takes advantage of an appropriate path integral representation for a kind of 4-point gauge invariant Green function and proceeds entirely from first principles apart from the so called modified area low assumption. Such assumption consists simply in adding an area term to the perturbative value of the logarithm of the Wilson loop correlator, and, as well known, it is well founded both on lattice simulation and on theoretical considerations

$$
i \ln W=i(\ln W)_{\text {pert }}+\sigma S_{\min } .
$$

Unfortunately a direct treatment of the full B-S equation in its four dimensional form seems to be beyond the present possibilities. From it, however, by a standard method (which includes replacement of the full quark propagator by a free propagator and an instantaneous approximation of the kernel) a center of mass hamiltonian can be obtained in the form

$$
H=\sqrt{m_{1}^{2}+\mathbf{k}^{2}}+\sqrt{m_{2}^{2}+\mathbf{k}^{2}}+V,
$$

with

$$
\begin{aligned}
& \left\langle\mathbf{k}|V| \mathbf{k}^{\prime}\right\rangle=\frac{1}{2 \sqrt{w_{1} w_{2} w_{1}^{\prime} w_{2}^{\prime}}}\left\{\frac { 4 } { 3 } \frac { \alpha _ { \mathrm { s } } } { \pi ^ { 2 } } \left[-\frac{1}{\mathbf{Q}^{2}}\left(q_{10} q_{20}+\mathbf{q}^{2}-\frac{(\mathbf{Q} \cdot \mathbf{q})^{2}}{\mathbf{Q}^{2}}\right)+\right.\right. \\
& +\frac{i}{2 \mathbf{Q}^{2}} \mathbf{k}^{\prime} \times \mathbf{k} \cdot\left(\boldsymbol{\sigma}_{1}+\boldsymbol{\sigma}_{2}\right)+\frac{1}{2 \mathbf{Q}^{2}}\left[q_{20}\left(\boldsymbol{\alpha}_{1} \cdot \mathbf{Q}\right)-q_{10}\left(\boldsymbol{\alpha}_{2} \cdot \mathbf{Q}\right)\right]+ \\
& \left.+\frac{1}{6} \boldsymbol{\sigma}_{1} \cdot \boldsymbol{\sigma}_{2}+\frac{1}{4}\left(\frac{1}{3} \boldsymbol{\sigma}_{1} \cdot \boldsymbol{\sigma}_{2}-\frac{\left(\mathbf{Q} \cdot \boldsymbol{\sigma}_{1}\right)\left(\mathbf{Q} \cdot \boldsymbol{\sigma}_{2}\right)}{\mathbf{Q}^{2}}\right)+\frac{1}{4 \mathbf{Q}^{2}}\left(\boldsymbol{\alpha}_{1} \cdot \mathbf{Q}\right)\left(\boldsymbol{\alpha}_{2} \cdot \mathbf{Q}\right)\right]+ \\
& \left.+\frac{1}{(2 \pi)^{3}} \int d^{3} \mathbf{r} e^{i \mathbf{Q} \cdot \mathbf{r}} J^{\text {inst }}\left(\mathbf{r}, \mathbf{q}, q_{10}, q_{20}\right)\right\}
\end{aligned}
$$

and

$$
\begin{aligned}
& J^{\mathrm{inst}}\left(\mathbf{r}, \mathbf{q}, q_{10}, q_{20}\right)=\frac{\sigma r}{q_{10}+q_{20}}\left[q_{20}^{2} \sqrt{q_{10}^{2}-\mathbf{q}_{\mathrm{T}}^{2}}+q_{10}^{2} \sqrt{q_{20}^{2}-\mathbf{q}_{\mathrm{T}}^{2}}+\right. \\
& \left.+\frac{q_{10}^{2} q_{20}^{2}}{\left|\mathbf{q}_{\mathrm{T}}\right|}\left(\arcsin \frac{\left|\mathbf{q}_{\mathrm{T}}\right|}{q_{10}}+\arcsin \frac{\left|\mathbf{q}_{\mathrm{T}}\right|}{q_{20}}\right)\right]-\frac{\sigma}{r}\left[\frac{q_{20}}{\sqrt{q_{10}^{2}-\mathbf{q}_{\mathrm{T}}^{2}}}\left(\mathbf{r} \times \mathbf{q} \cdot \boldsymbol{\sigma}_{1}+i q_{10}\left(\mathbf{r} \cdot \boldsymbol{\alpha}_{1}\right)\right)+\right. \\
& \left.+\frac{q_{10}}{\sqrt{q_{20}^{2}-\mathbf{q}_{\mathrm{T}}^{2}}}\left(\mathbf{r} \times \mathbf{q} \cdot \boldsymbol{\sigma}_{2}-i q_{20}\left(\mathbf{r} \cdot \boldsymbol{\alpha}_{2}\right)\right)\right] .
\end{aligned}
$$

In eq. (24) the perturbative term has been evaluated only at the first order in the coupling constant $\alpha_{\mathrm{s}}$, the indices 1 and 2 denote the quark and the antiquark, $\mathbf{k}$ and $\mathbf{k}^{\prime}$ denote the final and the initial center of mass momentum of the quark, $\sigma$ is the string tension, $\mathbf{q}=\frac{\mathbf{k}+\mathbf{k}^{\prime}}{2} ; \mathbf{Q}=\mathbf{k}^{\prime}-\mathbf{k} ; q_{j 0}=\frac{w_{j}+w_{j}^{\prime}}{2}, q_{\mathrm{T}}^{h}=\left(\delta^{h k}-\hat{r}^{h} \hat{r}^{k}\right) q^{k}$ is the transverse momentum, while $\alpha_{j}^{k}$ are the usual Dirac matrices $\gamma_{j}^{0} \gamma_{j}^{k}$, and $\sigma_{j}^{k}$ the $4 \times 4$ Dirac spin matrices $i / 4 \varepsilon^{k n m}\left[\gamma_{j}^{n}, \gamma_{j}^{m}\right]$.

It is shown in [罒 that by an $1 / m$ expansion and an appropriate Foldy-Wouthuysen transformation one arrives from eqs. (3) and (何) to the semirelativistic potential discussed 
in [2]. Such potential is made by a static part $V_{0}$, a velocity dependent part $V_{\mathrm{vd}}$ and a spin dependent part $V_{\text {sd }}$. The expression $V_{\text {sd }}$ is identical to that derived from the so called scalar confinement hypothesis while $V_{\mathrm{vd}}$ is different.

As shown in [2] and [3], when appropriate values are given to the quark masses $m_{j}$, to $\alpha_{\mathrm{s}}$ and to $\sigma$, the semirelativistic potential $V_{0}+V_{\mathrm{vd}}$ reproduces reasonably well the spin averaged multiplets in the heavy quarkonium case and it is phenomenologically favoured with respect to the scalar confinement expression. On the other hand it is shown in [5] that $V_{\text {sd }}$ gives the correct fine and hyperfine splittings if even the second order contributions in $\alpha_{\mathrm{s}}$ are included.

In this paper we want to treat directly the hamiltonian (2-1). In this way we shall see that we not only reproduce the same results for heavy quarkonia as from the semirelativistic potential, but we also obtain the correct ground Regge trajectories (with the right slope and intercepts) for the systems involving light quarks alone and the known lowest states for the systems with a light quark and a heavy antiquark. (On the contrary we did not succeed in evaluating daughter trajectories due to computation difficulties.) As we shall explain more precisely later, we have achieved this goal by a very small rearrangement of the parameters used in [3,4] and adopting current masses as given by the particle data group [6] for the light quarks $u, d$ and $s$.

Notice that to treat light-light and light-heavy systems various models have been attempted in literature, based on some conjectural choice of a B-S kernel (see for instance [7]) or directly of a local potential in eq. (2) (see e.g. [8] and references therein). However while such models give a reasonable spectrum, they usually fail in reproducing the observed Regge trajectories (in the sense that they do not result straight [7], or, if they do, they have not the correct slope and intercepts) for the values of the string tension required by the heavy quarkonia fit.

In this paper we shall neglect as a rule the spin dependent terms in eqs. (3) and (4) and shall mainly pay attention to the velocity dependent part alone. Indeed the inclusion of the spin-orbit terms in the light quark case would make the entire treatment much more involved due to the impossibility of an expansion in $1 / m$ and, as we shall see, it would not be particularly significant for our analysis. Later we shall return on S-wave hyperfine splitting which however would require the inclusion of the $\alpha_{\mathrm{s}}^{2}$ terms to give quantitative predictions. Finally in the light-light case we shall restrict our attention to triplet states alone. In fact for the light pseudoscalar mesons like $\pi, K, \eta, \eta^{\prime}$, the approximation of the quark propagators by their free expressions as implied in the use of an hamiltonian is certainly inadequate, due to the complicate interplay existing in this case between quark propagator and B-S wave function which is related to the chiral symmetry breaking [9].

Restricting our consideration to the velocity dependent part of the potential and splitting it in a perturbative and in a confinement part, $V=V_{\text {pert }}+V_{\text {conf }}$, we can write

$$
\left\langle\mathbf{k}\left|V_{\text {pert }}\right| \mathbf{k}^{\prime}\right\rangle=\frac{1}{2 \sqrt{w_{1} w_{2} w_{1}^{\prime} w_{2}^{\prime}}}\left\{-\frac{4}{3} \frac{\alpha_{\mathrm{s}}}{\pi^{2}}\left[\frac{1}{\mathbf{Q}^{2}}\left(q_{10} q_{20}+\mathbf{q}^{2}-\frac{(\mathbf{Q} \cdot \mathbf{q})^{2}}{\mathbf{Q}^{2}}\right)\right]\right\}
$$

and

$$
\left\langle\mathbf{k}\left|V_{\text {conf }}\right| \mathbf{k}^{\prime}\right\rangle=\frac{1}{2 \sqrt{w_{1} w_{2} w_{1}^{\prime} w_{2}^{\prime}}} \frac{1}{(2 \pi)^{3}} \int d^{3} \mathbf{r} e^{i \mathbf{Q} \cdot \mathbf{r}} J_{\text {si }}\left(\mathbf{r}, \mathbf{q}, q_{10}, q_{20}\right)
$$

with 


$$
\begin{array}{r}
J_{\mathrm{si}}\left(\mathbf{r}, \mathbf{q}, q_{10}, q_{20}\right)=\frac{\sigma r}{q_{10}+}+q_{20}\left[q_{20}^{2} \sqrt{q_{10}^{2}-\mathbf{q}_{\mathrm{T}}^{2}}+q_{10}^{2} \sqrt{q_{20}^{2}-\mathbf{q}_{\mathrm{T}}^{2}}+\right. \\
\left.+\frac{q_{10}^{2} q_{20}^{2}}{\left|\mathbf{q}_{\mathrm{T}}\right|}\left(\arcsin \frac{\left|\mathbf{q}_{\mathrm{T}}\right|}{q_{10}}+\arcsin \frac{\left|\mathbf{q}_{\mathrm{T}}\right|}{q_{20}}\right)\right] .
\end{array}
$$

In this way our hamiltonian becomes strictly related, but not identical, to that considered in ref. [10].

Let us begin to consider potential (5) and (6) in various different limit situations. First let us take the static limit consisting in setting $\mathbf{k}=\mathbf{k}^{\prime}=0$ in (5-7). In this way (5) and (6) become local and the hamiltonian can be written as

$$
H_{\text {stat }}=\sqrt{m_{1}^{2}+\mathbf{k}^{2}}+\sqrt{m_{2}^{2}+\mathbf{k}^{2}}-\frac{4}{3} \frac{\alpha_{\mathrm{s}}}{r}+\sigma r .
$$

Less drastically let us assume $\mathbf{q}_{\mathrm{T}}^{2} \ll \mathbf{q}^{2}$ (small angular momentum) and expand (7) in such quantity. Introducing the regularized Fourier transform

$$
\left\langle\mathbf{k}|\sigma r| \mathbf{k}^{\prime}\right\rangle=-\frac{\sigma}{\pi^{2}}\left(\frac{1}{\mathbf{Q}^{4}}\right)_{\mathrm{reg}}=\frac{\sigma}{\pi^{2}} \frac{1}{2} \frac{d^{2}}{d \varepsilon^{2}} \frac{1}{\mathbf{Q}^{2}+\varepsilon^{2}}
$$

(the limit $\varepsilon \rightarrow 0$ being understood) and using the identity

$$
\left\langle\mathbf{k}\left|r \mathbf{q}_{\mathrm{T}}^{2}\right| \mathbf{k}^{\prime}\right\rangle=\left\langle\mathbf{k}\left|\frac{L^{2}+1}{r}\right| \mathbf{k}^{\prime}\right\rangle
$$

$L^{2}$ being the orbital angular momentum of the two particles (notice that classically $\mathbf{q}_{\mathrm{T}}^{2}=$ $\left.\mathbf{k}_{\mathrm{T}}^{2}=\frac{L^{2}}{r^{2}}\right)$, one finds

$$
\begin{gathered}
\left\langle\mathbf{k}\left|V_{\text {conf }}\right| \mathbf{k}^{\prime}\right\rangle=-\frac{\sigma}{2 \pi^{2}} \frac{1}{\sqrt{w_{1}^{\prime} w_{2}^{\prime} w_{1} w_{2}}}\left[2 q_{10} q_{20}\left(\frac{1}{\mathbf{Q}^{4}}\right)_{\mathrm{reg}}+\right. \\
\left.+\frac{1}{6} \frac{1}{q_{10}+q_{20}}\left(\frac{q_{10}^{2}}{q_{20}}+\frac{q_{20}^{2}}{q_{10}}\right)\left\langle\mathbf{k}\left|L^{2}+1\right| \mathbf{k}^{\prime}\right\rangle \frac{1}{\mathbf{Q}^{2}}+\cdots\right] .
\end{gathered}
$$

On the contrary setting in eq. (7) $\mathbf{q}_{\mathrm{T}}^{2} \simeq \mathbf{q}^{2}$ (large angular momentum) one has

$$
\begin{aligned}
\left\langle\mathbf{k}\left|V_{\text {conf }}\right| \mathbf{k}^{\prime}\right\rangle= & -\frac{\sigma}{2 \pi^{2}} \frac{1}{\sqrt{w_{1}^{\prime} w_{2}^{\prime} w_{1} w_{2}}} \frac{1}{q_{10}+q_{20}}\left(\frac{1}{\mathbf{Q}^{4}}\right)_{\text {reg }}\left[q_{20}^{2} \sqrt{q_{10}^{2}-\mathbf{q}^{2}}+\right. \\
& \left.+q_{10}^{2} \sqrt{q_{20}^{2}-\mathbf{q}^{2}}+\frac{q_{10}^{2} q_{20}^{2}}{|\mathbf{q}|}\left(\arcsin \frac{|\mathbf{q}|}{q_{10}}+\arcsin \frac{|\mathbf{q}|}{q_{20}}\right)\right] .
\end{aligned}
$$

Finally if in eq. (12) we consider the extreme case $m_{1}=m_{2}=0$, neglect $V_{\text {pert }}$ and simply set $\mathbf{k}=\mathbf{k}^{\prime}$ in the factor multiplying the singular term

$$
\frac{1}{\mathrm{Q}^{4}}=\frac{1}{\left(\mathbf{k}^{\prime}-\mathbf{k}\right)^{4}}
$$

we obtain the hamiltonian 


$$
H_{\mathrm{hqt}}=2|\mathbf{q}|+\frac{\pi}{4} \sigma r
$$

In literature eq. (8) has been used as such. In ref. 8], e.g. an overall fit of the low angular momentum meson spectrum has been obtained by setting $\sigma=0.22 \mathrm{GeV}^{2}, \alpha_{\mathrm{s}}=0.323$ and adding to the lefthand of eq. (8) an ad hoc flavour dependent constant $C$ in the sector of the light quarks. As well known, however, for small $m_{1}$ and $m_{2}$ eq. (8) would give asymptotic straight Regge trajectories with slope $\alpha^{\prime}=1 / 8 \sigma$, which for $\sigma=0.22 \mathrm{GeV}^{2}$ gives $\alpha^{\prime}=0.57$ $\mathrm{GeV}^{-2}$ while the experimental value is about $\alpha^{\prime}=0.88 \mathrm{GeV}^{-2}$.

On the contrary from eq. (14) we would obtain

$$
\alpha^{\prime}=\frac{1}{8 \frac{\pi}{4} \sigma}=\frac{1}{2 \pi \sigma}
$$

which is identical to the Nambu-Goto string model. This equation gives the correct experimental value for $\sigma \simeq 0.18 \mathrm{GeV}^{2}$, which is the value used e.g. in ref. [3], 1] to fit the $c \bar{c}$ and the $b \bar{b}$ spectra (when the coupling with the decay channels is neglected). This result illustrates the advantage of considering the potential (56) with respect to a simply local potential of the type appearing in (8).

Actually we do not succeed in directly diagonalizing the hamiltonian eqs. (2,55-7), due to problems of numerical stability and computer time. Therefore we follow the following strategy. First we diagonalize the static hamiltonian $H_{\text {stat }}$ by the Rayleigh-Ritz variational method using the harmonic oscillator wavefunctions as basis [11, 12]. These wavefunctions in coordinate space take the form

$$
\phi_{n l m}(\lambda, \mathbf{r})=\Phi_{n l}\left(\frac{r}{\lambda}\right) Y_{l m}(\hat{r})=\frac{1}{\pi^{\frac{1}{4}}} \frac{1}{\lambda^{\frac{3}{2}}} \sqrt{\frac{2^{n+l+1}(n-1) !}{[2(n+l)-1] ! !}}\left(\frac{r}{\lambda}\right)^{l} L_{n-1}^{\left(l+\frac{1}{2}\right)}\left(\frac{r^{2}}{\lambda^{2}}\right) e^{-\frac{r^{2}}{2 \lambda^{2}}} Y_{l m}(\hat{r})
$$

and in the momentum space

$$
\begin{aligned}
\phi_{n l m}(\lambda, \mathbf{k}) & =\Phi_{n l}(\lambda k) Y_{l m}(\hat{k})= \\
& =\frac{(-1)^{n-1}}{\pi^{\frac{1}{4}}} \lambda^{\frac{3}{2}}(-i)^{l} \sqrt{\frac{2^{n+l+1}(n-1) !}{[2(n+l)-1] ! !}}(\lambda k)^{l} L_{n-1}^{\left(l+\frac{1}{2}\right)}\left(\lambda^{2} k^{2}\right) e^{-\frac{\lambda^{2} k^{2}}{2}} Y_{l m}(\hat{k}),
\end{aligned}
$$

where $\lambda$ is a scale factor and $L_{n-1}^{\left(l+\frac{1}{2}\right)}$ are the Laguerre polynomials [13. Then we write the eigenfunctions of $H_{\text {stat }}$ as

$$
\psi_{n l m}(\lambda, \mathbf{k})=\sum_{n^{\prime}} a_{n n^{\prime}}^{l} \phi_{n^{\prime} l m}(\lambda, \mathbf{k})=\Psi_{n l}(\lambda k) Y_{l m}(\hat{k})
$$

and evaluate the expectation value

$$
\left\langle\psi_{n l m}|H| \psi_{n l m}\right\rangle \text {. }
$$

Finally the eigenvalues of $H$ are estimated as the minima of (19) in $\lambda$.

Due to eq. (17) the evaluation of the kinetic term in eq. (19) is simply reduced to a one dimensional integral.

For the perturbative term, by setting 


$$
z_{0}=\frac{k^{\prime 2}+k^{2}}{2 k^{\prime} k}, \quad z_{\varepsilon}=\frac{k^{2}+k^{2}+\varepsilon^{2}}{2 k^{\prime} k}
$$

we obtain from eq. (5)

$$
\begin{array}{r}
\left\langle\psi_{n l m}\left|V_{\text {pert }}\right| \psi_{n l m}\right\rangle=-\frac{4}{3} \frac{\alpha_{\mathrm{s}}}{\pi} \frac{1}{2} \int_{0}^{\infty} d k^{\prime} k^{\prime} \frac{1}{\sqrt{w_{1}^{\prime} w_{2}^{\prime}}} \Psi_{n l}^{*}\left(\lambda k^{\prime}\right) \int_{0}^{\infty} d k k \frac{1}{\sqrt{w_{1} w_{2}}} \Psi_{n l}(\lambda k) \\
\left\{\left(2 q_{10} q_{20}+\frac{k^{\prime 2}+k^{2}}{2}\right) Q_{l}\left(z_{0}\right)+\frac{k^{\prime} k}{2 l+1}\left[(l+1) Q_{l+1}\left(z_{0}\right)+l Q_{l-1}\left(z_{0}\right)\right]+\right. \\
\left.+\frac{\left(k^{\prime}-k\right)^{2}\left(k^{\prime}+k\right)^{2}}{4 k^{\prime} k}\left[\left(\frac{d}{d z_{0}} P_{l}\left(z_{0}\right)\right) \ln \left|\frac{k^{\prime}+k}{k^{\prime}-k}\right|-\frac{d}{d z_{0}} W_{l-1}\left(z_{0}\right)\right]-k^{\prime} k P_{l}\left(z_{0}\right)\right\}
\end{array}
$$

( $P_{l}$ and $Q_{l}$ being respectively the first and second kind Legendre polynomials) a double integral.

For what concerns $\left\langle\psi_{n l m}\left|V_{\text {conf }}\right| \psi_{n l m}\right\rangle$, if we had used the original expression (6, (7), we should have ended in a five-dimensional integral which numerical evaluation is quite problematic for a strongly oscillating function like $\psi_{n l m}(\lambda, \mathbf{k})$. For this reason in place of (6) and (घ) we take in turn advantage of the approximate eqs. (11) or (12). From eq. (11) we obtain

$$
\begin{array}{r}
\left\langle\psi_{n l m}\left|V_{\text {conf }}\right| \psi_{n l m}\right\rangle=\frac{\sigma}{2 \pi} \int_{0}^{\infty} d k^{\prime} k^{\prime} \frac{1}{\sqrt{w_{1}^{\prime} w_{2}^{\prime}}} \Psi_{n l}^{*}\left(\lambda k^{\prime}\right) \int_{0}^{\infty} d k k \frac{1}{\sqrt{w_{1} w_{2}}} \Psi_{n l}(\lambda k) \\
{\left[2 q_{10} q_{20} \lim _{\varepsilon \rightarrow 0} \frac{d^{2}}{d \varepsilon^{2}} Q_{l}\left(z_{\varepsilon}\right)-\frac{1}{3} \frac{1}{q_{10}+q_{20}}\left(\frac{q_{10}^{2}}{q_{20}}+\frac{q_{20}^{2}}{q_{10}}\right)\left(L^{2}+1\right) Q_{l}(z)\right]}
\end{array}
$$

(again a double integral) and from eq. (12)

$$
\begin{aligned}
\left\langle\psi_{n l m}\left|V_{\text {conf }}\right| \psi_{n l m}\right\rangle= & \frac{\sigma}{2 \pi} \int_{0}^{\infty} d k^{\prime} k^{\prime 2} \frac{1}{\sqrt{w_{1}^{\prime} w_{2}^{\prime}}} \Psi_{n l}^{*}\left(\lambda k^{\prime}\right) \int_{0}^{\infty} d k k^{2} \frac{1}{\sqrt{w_{1} w_{2}}} \Psi_{n l}(\lambda k) \frac{1}{q_{10}+q_{20}} \\
& \int_{-1}^{1} d \xi P_{l}(\xi) \lim _{\varepsilon \rightarrow 0} \frac{d^{2}}{d \varepsilon^{2}} \frac{1}{\mathbf{Q}^{2}+\varepsilon^{2}}\left[q_{20}^{2} \sqrt{q_{10}^{2}-\mathbf{q}^{2}}+q_{10}^{2} \sqrt{q_{20}^{2}-\mathbf{q}^{2}}+\right. \\
& \left.+\frac{q_{10}^{2} q_{20}^{2}}{|\mathbf{q}|}\left(\arcsin \frac{|\mathbf{q}|}{q_{10}}+\arcsin \frac{|\mathbf{q}|}{q_{20}}\right)\right]
\end{aligned}
$$

(a triple integral). The singularities occurring in the integrals in eqs. (21) and (22) or (23) can be handled by the method explained in ref. [14].

We use eq. (22) for the evaluation of the heavy quarkonia spectrum and for the light quarks S state, eq. (23) for the Regge trajectories.

Notice that eq. (22) produces systematically larger masses. The difference being of the order of few $\mathrm{MeV}$ for the $b \bar{b}$ system, between 5 and $15 \mathrm{MeV}$ for $c \bar{c}$ and progressively larger if light quarks are involved. The subsequent term in expansion (22) would contain a $L^{2}\left(L^{2}+2\right)$ factor and the convergence of the expansion soon becomes very slow as $L$ increases. For this reason we belive eq. (23) to be preferred for light quarks already for the P states.

Once that the spin averaged masses are determined we may evaluate the $\mathrm{S}$-wave hyperfine splitting by the equation 


$$
\Delta=\left\langle\psi_{n l m}\left|V_{3}-V_{1}\right| \psi_{n l m}\right\rangle=\frac{4}{3 \pi} \frac{4}{3} \alpha_{\mathrm{s}} \delta_{l 0}\left[\int_{0}^{\infty} d k \frac{k^{2}}{\sqrt{w_{1} w_{2}}} \Psi_{n l}(\lambda k)\right]^{2}
$$

this is obtained taking into consideration only the pure $\boldsymbol{\sigma}_{1} \cdot \boldsymbol{\sigma}_{2}$ term in eq. (33).

We have adopted the following parameters: $\alpha_{\mathrm{s}}=0.363, \sigma=0.175 \mathrm{GeV}^{2}, m_{c}=1.40$ $\mathrm{GeV}, m_{b}=4.81 \mathrm{GeV}, m_{s}=200 \mathrm{MeV}, m_{u}=10 \mathrm{MeV}$; no ad hoc constant $C$ has been added to the potential. The first four values have to be compared with those obtained from heavy quarkonium fits. E.g. when pair creation effects are neglected (and after renormalization of the masses by reabsorbing the constant $C$ ) the values used in ref. [3] (table 2, first column) become $\alpha_{\mathrm{s}}=0.363, \sigma=0.178 \mathrm{GeV}^{2}, m_{c}^{\prime}=m_{c}+\frac{C}{2}=1.397 \mathrm{GeV}, m_{b}^{\prime}=m_{b}+\frac{C}{2}=4.792$ $\mathrm{GeV}$, (cf. also ref. [10] fig. 4). On the contrary the light quark masses are taken as the baricentral values of the current masses: $m_{u}^{\text {current }}=2$ to $8 \mathrm{MeV}, m_{d}^{\text {current }}=5$ to $15 \mathrm{MeV}$, $m_{s}^{\text {current }}=100$ to $300 \mathrm{MeV}$ as reported from the Particle Data Group [6]. Notice also in this connection $m_{c}^{\text {current }}=1.0$ to $1.6 \mathrm{GeV}, m_{b}^{\text {current }}=4.1$ to $4.5 \mathrm{GeV}$. The small rearrangement in the values of $\sigma, m_{c}$ and $m_{b}$ is required to obtain the exact slope for the Regge trajectories without modifying the $\psi$ and the $\Upsilon$ ground states. Notice that apart from that no attempt of optimizing the parameter is made.

The results of the calculation for the $c \bar{c}, b \bar{b}$ systems are reported in table $\mathbb{q}$ and compared with the spin averaged multiplets. An estimate for such values is taken from ref. [8]. Also the unperturbed values obtained by $H_{\text {stat }}$ are reported, as it can be seen the shift for heavy quarks is of some tenth $\mathrm{MeV}$ (but such shift becomes more important for light quarks).

As expected we have reasonable overall agreement with the data, the discrepancies being in part ascribable to pair creation effects [3].

In Fig. 17, 2, 3 the Regge trajectories are reported for the ground triplet states of the systems $u \bar{u}, s \bar{s}, u \bar{s}$ corresponding to $J=L+1, L, L-1$. The agreement is again usually very good, particularly for the states $J=L+1$, while some disagreement, particularly in the $u \bar{s}$ case, can possibly be retraced in the neglecting of the spin-orbit terms. In table [1] numerical results are reported for the $u \bar{u}$ system and compared with the data for the $J=L+1$ state. The values in bracket for the $1 \mathrm{~S}$ and $2 \mathrm{~S}$ states are obtained by adding $1 / 4$ of the hyperfine splitting as given in table IV. Notice that the $2 \mathrm{~S}$ state of table 1 is too high by about 100 $\mathrm{MeV}$, such discrepancy is however smaller than the width of $\rho(1450)$ and $\omega(1420)$ and again could be ascribable to pair creation effects. Similar circumstances occur for the $u \bar{s}$ and $s \bar{s}$ cases.

The results for $u \bar{c}, u \bar{b}, s \bar{c}$, and $s \bar{b}$ systems are reported in table 【II and compared with the experimental spin averaged masses using the theoretical splitting when the singlet state has not yet been observed.

In table $\square$ the hyperfine splittings as evaluated by eq. (24) are reported for the heavyheavy and the heavy-light cases. In the light-light case a comparison with the splitting $\rho-\pi$, $\varphi-\eta^{\prime}$ and $K^{*}-K$ would have no meaning for the reason we have explained. Notice however that for the $u \bar{u}$ ground state eq. (24) would give $\Delta=221 \mathrm{MeV}$ in good agreement with the $\omega-\eta$ splitting $(234 \mathrm{MeV})$.

The fact that we do not have to use constituent masses in our calculation seem surprising at first sight. Notice however that, following [15], if we expand the kinetic part of the hamiltonian eq. (2) around the expectation value $\left\langle\psi_{n l m}\left|\mathbf{k}^{2}\right| \psi_{n l m}\right\rangle$ (that we call simply $\left\langle\mathbf{k}^{2}\right\rangle$ ) we find 


$$
M=m_{1 \mathrm{eff}}+m_{2 \mathrm{eff}}+\frac{\mathbf{k}^{2}}{2 m_{1 \mathrm{eff}}}+\frac{\mathbf{k}^{2}}{2 m_{2 \mathrm{eff}}}-\frac{\mathbf{k}^{4}}{8 m_{1 \mathrm{eff}}^{3}}-\frac{\mathbf{k}^{4}}{8 m_{2 \mathrm{eff}}^{3}}+V+C
$$

where at this order

$$
m_{i \text { eff }}=\sqrt{m_{i}^{2}+\left\langle\mathbf{k}^{2}\right\rangle}\left(1+\frac{1}{2} \frac{\left\langle\mathbf{k}^{2}\right\rangle}{m_{i}^{2}+\left\langle\mathbf{k}^{2}\right\rangle}\right)^{-1}, \quad i=1,2
$$

and the quantity $C$ can be defined by difference.

Average values of $m_{u \text { eff }}$ and $m_{s \text { eff }}$ for the lowest states involving quarks $u$ and $s$ obtained evaluating $\left\langle\mathbf{k}^{2}\right\rangle$ for the wave function eq. (18) are typically $m_{u \text { eff }}=360 \mathrm{MeV}, m_{s \text { eff }}=410$ $\mathrm{MeV}, m_{c \text { eff }}=1.440 \mathrm{GeV}, m_{b \text { eff }}=4.820 \mathrm{GeV}$, with $C \simeq 300 \mathrm{MeV}$. These are of the same order of the constituent masses used in semirelativistic computations [15].

In conclusion, starting from our first principle Salpeter equation, we have obtained an overall good reproduction of the spectrum of the mesons involving heavy and light quarks with the exception of the light pseudoscalar states. Since the parameter are practically completely specified by the heavy quarkonium spectrum and by high energy scattering, our calculation is essentially parameter free, for what concerns light-light and light-heavy quark systems.

We would like to thank K. Maung Maung, L. Sorrillo and R. Chen for the useful discussions and N. Brambilla and A. Vairo for assistance on numerical problems. 


\section{TABLES}

TABLE I. Spin averaged energies for heavy quarkonium systems. Our theoretical results and experimental values.

\begin{tabular}{cccc}
\hline \hline States & $\begin{array}{c}\text { static potential } \\
(\mathrm{GeV})\end{array}$ & $\begin{array}{c}\text { total potential } \\
(\mathrm{GeV})\end{array}$ & $\begin{array}{c}\text { experimental values } \\
(\mathrm{GeV})\end{array}$ \\
\hline$b \bar{b}$ & & & \\
$1 \mathrm{~S}$ & 9.504 & 9.446 & $9.448 \pm 0.005$ \\
$2 \mathrm{~S}$ & 10.055 & 10.014 & $10.017 \pm 0.005$ \\
$3 \mathrm{~S}$ & 10.385 & 10.347 & $10.351 \pm 0.005$ \\
$4 \mathrm{~S}$ & 10.652 & 10.615 & $10.580 \pm \quad ?$ \\
$5 \mathrm{~S}$ & 10.886 & 10.849 & $10.865 \pm ?$ \\
$6 \mathrm{~S}$ & 11.109 & 11.068 & $11.019 \pm ?$ \\
$1 \mathrm{P}$ & 9.953 & $9.900 \pm 0.001$ \\
$2 \mathrm{P}$ & 9.972 & 10.288 & $10.260 \pm 0.001$ \\
\hline$c \bar{c}$ & 10.310 & & \\
$1 \mathrm{~S}$ & & 3.065 & $3.067 \pm 0.002$ \\
$2 \mathrm{~S}$ & 3.133 & 3.623 & $3.663 \pm 0.005$ \\
$3 \mathrm{~S}$ & 3.693 & 4.030 & $4.040 \pm ?$ \\
$4 \mathrm{~S}$ & 4.100 & 4.372 & $4.415 \pm ?$ \\
$1 \mathrm{P}$ & 4.441 & 3.508 & $3.525 \pm 0.001$ \\
$1 \mathrm{D}$ & 3.551 & 3.794 & $3.770 \pm \quad ?$ \\
$2 \mathrm{D}$ & 3.833 & 4.158 & $4.159 \pm ?$ \\
\hline \hline
\end{tabular}


TABLE II. Theoretical results and experimental data for $u \bar{u}$ system. For the S states we write the theoretical averaged mass and in brackets the mass for the triplet state obtained with the hyperfine splitting collected in table IV.

\begin{tabular}{|c|c|c|c|}
\hline \multirow[t]{2}{*}{$\overline{\text { State }}$} & \multirow{2}{*}{$\begin{array}{c}\text { M theor. } \\
(\mathrm{GeV})\end{array}$} & \multicolumn{2}{|c|}{ Exper. data $(\mathrm{GeV})$} \\
\hline & & $\omega \quad I^{G}\left(J^{P C}\right)$ & $\rho \quad I^{G}\left(J^{P C}\right)$ \\
\hline \multirow[t]{2}{*}{$\overline{1^{3} \mathrm{~S}_{1}}$} & 0.740 & $\omega(782) 0^{-}\left(1^{--}\right)$ & $\rho(770) 1^{+}\left(1^{--}\right)$ \\
\hline & $(0.795)$ & $0.78194 \pm 0.00012$ & $0.7685 \pm 0.0006$ \\
\hline \multirow[t]{2}{*}{$\overline{2^{3} \mathrm{~S}_{1}}$} & 1.543 & $\omega(1420) 0^{-}\left(1^{--}\right)$ & $\rho(1450) 1^{+}\left(1^{--}\right)$ \\
\hline & $(1.556)$ & $1.419 \pm 0.031$ & $1.465 \pm 0.025$ \\
\hline \multirow[t]{2}{*}{$\overline{1^{3} \mathrm{P}_{2}}$} & 1.323 & $f_{2}(1270) 0^{+}\left(2^{++}\right)$ & $a_{2}(1320) 1^{-}\left(2^{++}\right)$ \\
\hline & & $1.275 \pm 0.005$ & $1.3181 \pm 0.0007$ \\
\hline \multirow[t]{2}{*}{$1^{3} \mathrm{D}_{3}$} & 1.721 & $\omega_{3}(1667) 0^{-}\left(3^{--}\right)$ & $\rho_{3}(1690) 1^{+}\left(3^{--}\right)$ \\
\hline & & $1.667 \pm 0.004$ & $1.691 \pm 0.005$ \\
\hline \multirow[t]{2}{*}{$\overline{1^{3} \mathrm{~F}_{4}}$} & 2.031 & $f_{4}(2050) 0^{+}\left(4^{++}\right)$ & $a_{4}(2040) 1^{-}\left(4^{++}\right)$ \\
\hline & & $2.044 \pm 0.011$ & $2.037 \pm 0.026$ \\
\hline \multirow[t]{2}{*}{$1^{3} \mathrm{G}_{5}$} & 2.296 & & $\rho_{5}(2350) 1^{+}\left(5^{--}\right)$ \\
\hline & & & $2.330 \pm 0.035$ \\
\hline \multirow[t]{2}{*}{$\overline{1^{3} \mathrm{H}_{6}}$} & 2.531 & $f_{6}(2510) 0^{+}\left(6^{++}\right)$ & $a_{6}(2450) 1^{-}\left(6^{++}\right)$ \\
\hline & & $2.510 \pm 0.030$ & $2.450 \pm 0.130$ \\
\hline
\end{tabular}

TABLE III. Theoretical results for $u \bar{c}, u \bar{b}, s \bar{c}, s \bar{b}$ systems (MeV). Experimental data are enclosed in brackets.

\begin{tabular}{lcccc}
\hline \hline State & $u \bar{c}$ & $u \bar{b}$ & $s \bar{c}$ & $s \bar{b}$ \\
\hline $1 \mathrm{~S}$ & $1973(1973 \pm 1)$ & $5326(5313 \pm 2)$ & $2080(2076.4 \pm 0.5)$ & $5418(5404.6 \pm 2.5)$ \\
$2 \mathrm{~S}$ & $2600(2623 \pm ?)^{\mathrm{a}}$ & $5906(5897 \pm ?)^{\mathrm{a}}$ & & \\
$1 \mathrm{P}$ & $2442(2438 \pm ?)^{\mathrm{b}}$ & $5777(5825 \pm 14)^{\mathrm{c}}$ & $2528(2535.35 \pm 0.34)$ & $5848(5853 \pm 15)$ \\
\hline \hline
\end{tabular}

abtained from preliminary Delphi data $m\left(D^{* \prime}\right)=2637 \pm 8 \mathrm{MeV}, m\left(B^{* \prime}\right)=5906 \pm 14 \mathrm{MeV}$ [16] subtracting $1 / 4$ theoretical hyperfine splitting reported in table IV.

${ }^{\mathrm{b}}$ Estimated from $m\left(D_{2}^{*}\right)=2459 \pm 4 \mathrm{MeV}, m\left(D_{1}\right)=2427 \pm 5 \mathrm{MeV}$.

${ }^{\mathrm{c}}$ From preliminary Delphi data [16].

TABLE IV. Theoretical results for $q \bar{q}$ hyperfine splitting $(\mathrm{MeV})$. Experimental data are enclosed in brackets.

\begin{tabular}{lcccccc}
\hline \hline State & $u \bar{c}$ & $u \bar{b}$ & $c \bar{c}$ & $b \bar{b}$ & $s \bar{c}$ & $s \bar{b}$ \\
\hline $1 \mathrm{~S}$ & $111(141 \pm 1)$ & $59(46 \pm 3)$ & $97(117 \pm 2)$ & 102 & $108(144)$ & $60(47 \pm 4)$ \\
$2 \mathrm{~S}$ & 59 & 38 & $59(92 \pm 5)$ & 42 & 62 & 40 \\
\hline \hline
\end{tabular}




\section{FIGURE CAPTIONS}

Fig. 1

Ground triplet $u \bar{u}$ Regge trajectories. Theoretical results (full line) compared with experimental data (circlet). Cross denote less established masses.

Fig. 2

Ground triplet $s \bar{s}$ Regge trajectories (with the same notations of fig. 1).

Fig. 3

Ground triplet $u \bar{s}$ Regge trajectories (with the same notations of fig. 1). 


\section{FIGURES}

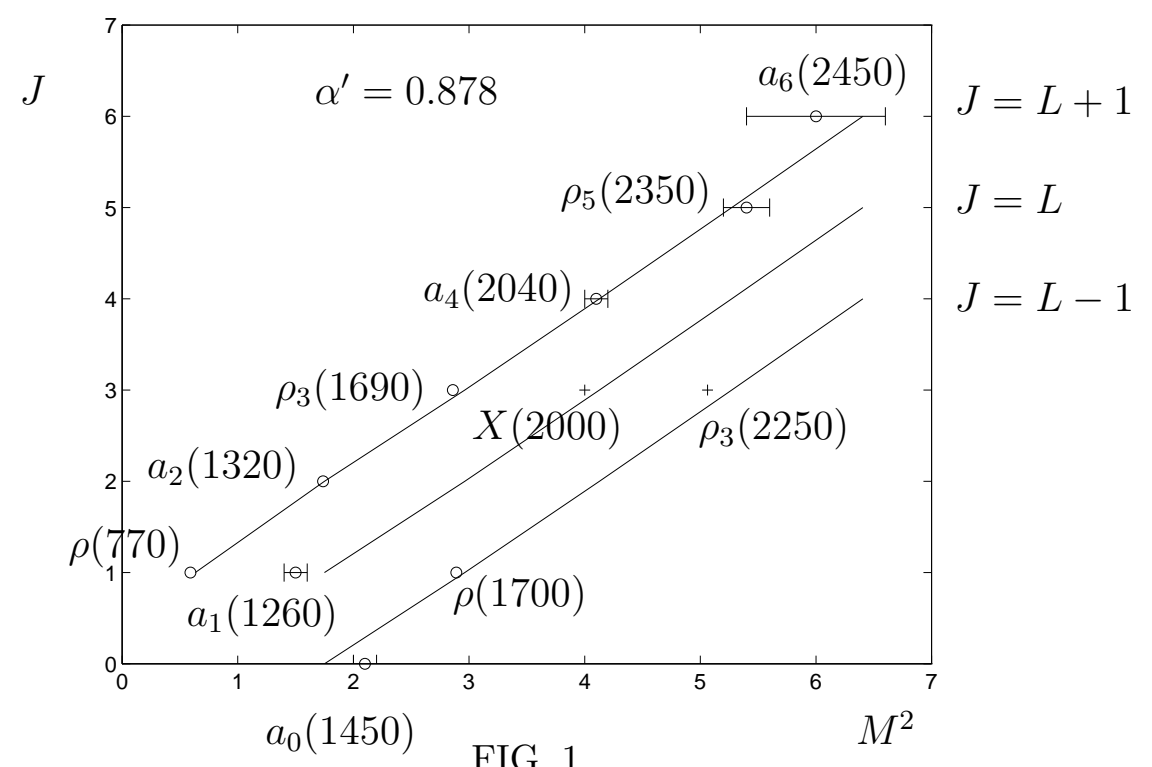

FIG. 1.

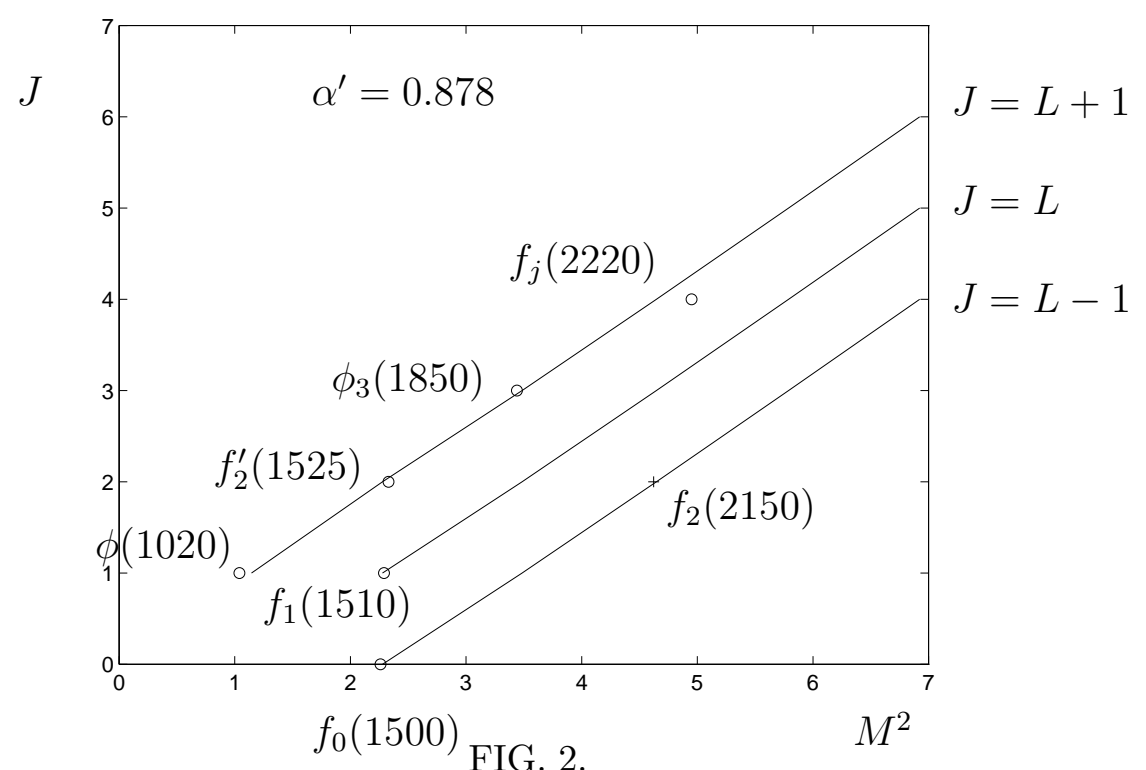




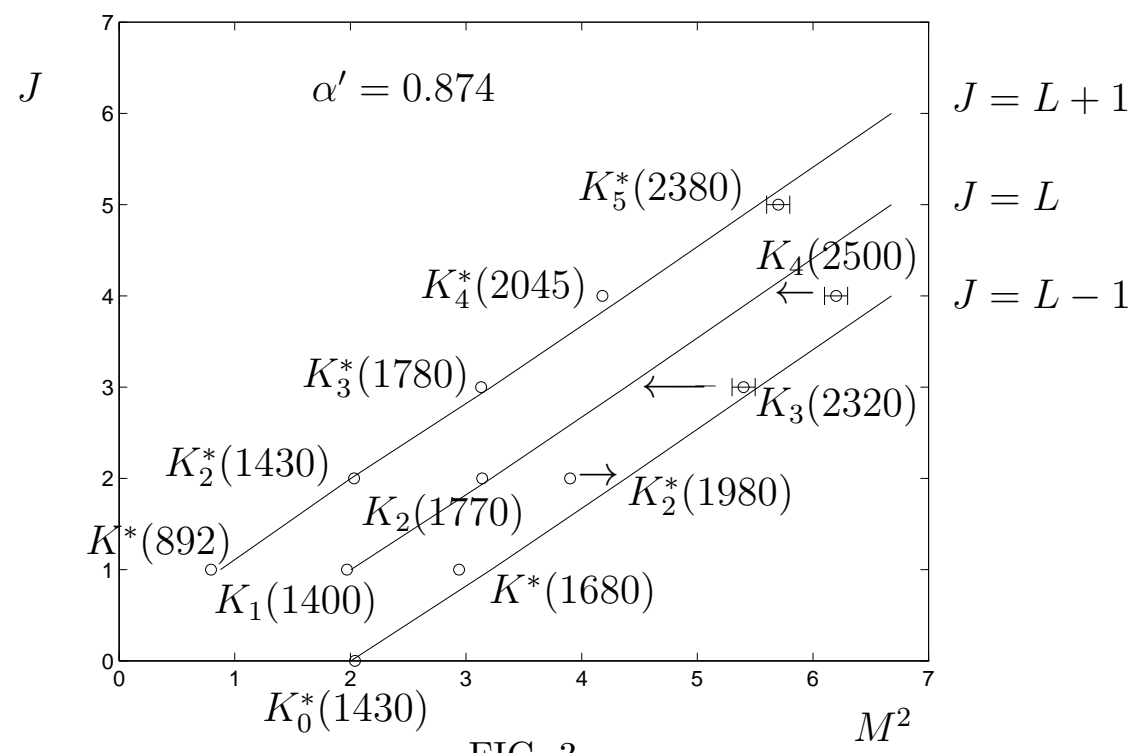

FIG. 3. 


\section{REFERENCES}

[1] N. Brambilla, E. Montaldi and G.M. Prosperi, Phys. Rev. D 54 (1996) 3506.

[2] N. Brambilla and G.M. Prosperi, in Quark Confinement and the Hadron Spectrum, edited by N. Brambilla and G.M. Prosperi (World Scientific, Singapore, 1995), p. 113 and references therein.

[3] N. Brambilla and G.M. Prosperi, Phys. Lett. B 236 (1990) 69.

[4] A. Barchielli, N. Brambilla and G.M. Prosperi, Il Nuovo Cimento 103 A (1990) 59.

[5] F. Halzen, C. Olson, M.G. Olsson, and M.L. Stong, Phys. Rev. D 47 (1993) 3013; S.N. Gupta and S.F. Radford, Phys. Rev. D 24 (1981) 2309.

[6] Particle Data Group, R.M. Barnett et al., Phys. Rev. D 54 (1996).

[7] A. Gara, B. Durand, L Durand and L.J. Nickisch, Phys. Rev. D 40 (1989) 843.

[8] L.P. Fulcher, Phys. Rev. D 50 (1994) 447.

[9] C.D. Roberts and B.H.J. McKellar, Phys. Rev. D 41 (1990) 672;

G.M. Prosperi, hep-th/9709046.

[10] M.G. Olsson, in Quark Confinement and the Hadron Spectrum, edited by N. Brambilla and G.M. Prosperi (World Scientific, Singapore, 1994), p. 76 and references therein.

[11] W. Lucha and F.F. Schöberl, Phys. Rev. A 54 (1996) 3790;

W. Lucha and F.F. Schöberl, Phys. Lett. B 387 (1996) 573;

W. Lucha and F.F. Schöberl, Phys. Rev. A 56 (1997) 139.

[12] M. Reed and B. Simon, Methods of Modern Mathematical Physics IV: Analysis of Operators (Academic Press, New York, 1978) Section XIII.1 and XIII.2

[13] E.J. Weniger, J. Math. Phys. 26 (2) (1985) 276.

[14] J.W. Norbury, D.E. Kahana and K.Maung Maung, Can. J.Phys. 70 (1992) 86; K.Maung Maung, D.E. Kahana and J.W. Norbury, Phys. Rev. D 47 (1993) 1182; R. Chen, L. Sorrillo and K. Maung Maung, preprint.

[15] A. Martin, Comments Nucl. Part. Phys. 16 (1986) 249;

W. Lucha, F.F. Schöberl and D. Gromes, Phys. Rep. 200 (1991) 127;

W. Lucha and F.F. Schöberl, Int. J. Mod. Phys. A7 (1992) 6431;

J.L. Basdevant and S. Boukraa, Z. Phys. C, 28 (1985) 413; 30 (1986) 103;

A.M. Badalyan, D.I. Kitoroage and D.S. Parĩskĩ, Sov. J. Nucl. Phys. 46 (1), (1987) 139; A.M. Badalyan and D.I. Kitoroage, ibid. 47 (3) (1988) 515.

[16] A. Pullia (private communication). 